\title{
Laser-Induced Optical Breakdown in Methane: Diagnostic Using H-Gamma Line Broadening
}

\author{
Christian G. Parigger ${ }^{1}$ and Eugene Oks ${ }^{2}$ \\ ${ }^{1}$ Center for Laser Applications, The University of Tennessee Space Institute, 411 B.H. Goethert Parkway, Tullahoma, TN 37388, USA \\ 2206 Allison Laboratory, Department of Physics, Auburn University, Auburn, AL 36849, USA
}

Correspondence should be addressed to Christian G. Parigger, cparigge@utsi.edu

Received 6 April 2009; Accepted 8 June 2009

Academic Editor: Elisabeth Dalimier

Copyright ( 2010 C. G. Parigger and E. Oks. This is an open access article distributed under the Creative Commons Attribution License, which permits unrestricted use, distribution, and reproduction in any medium, provided the original work is properly cited.

\begin{abstract}
Measurements and analysis are reported of Stark-broadened profiles of the H-gamma line emitted from plasma formed by LaserInduced Optical Breakdown (LIOB) in a pulsed methane flow. Electron densities $N_{e}$ are deduced for 18 instants of time in the range between 0.4 and 2.1 microseconds after LIOB and for 2 different gas pressures. The obtained values of $N_{e}$ are in a good agreement with the corresponding values found previously from Stark-broadened profiles of the $\mathrm{H}$-alpha and $\mathrm{H}$-beta lines.
\end{abstract}

\section{Introduction}

Plasmas formed by a Laser-Induced Optical Breakdown (LIOB) in gases can be diagnosed using the Stark broadening of hydrogen lines. In papers $[1,2]$, the LIOB was studied in gaseous hydrogen, and the plasmas were diagnosed using $\mathrm{H}$ alpha line in [1] and H-beta line in [2].

In the latest paper [3], the LIOB was studied in a pulsed methane flow; the plasma was diagnosed using timeresolved measurements of profiles of $\mathrm{H}$-alpha, $\mathrm{H}$-beta, and $\mathrm{H}$-gamma-lines. However, in [3] the electron density was deduced from the Stark broadening of only $\mathrm{H}$-alpha and $\mathrm{H}$ beta lines, while the measured widths of the H-gamma line have not been analyzed.

In the present paper we analyze the widths of the $\mathrm{H}$ gamma line measured in [3]. For 18 instants of time in the range between 0.4 and $2.1 \mu$ s after the LIOB and for 2 different gas pressures, we deduce the electron density $N_{e}$ from the Stark broadening of the H-gamma line and compare the results with the corresponding values of $N_{e}$ obtained from the H-alpha and H-beta lines.

\section{Experimental Results}

The time-resolved measurements of LIOB comprised typical experimental arrangements for laser-induced breakdown spectroscopy (LIBS): nominal nanosecond Q-switched laser $(8 \mathrm{~ns}, 75 \mathrm{~mJ}$ per pulse infrared $1064 \mathrm{~nm}$ radiation from a model Continuum YG680-10 Nd:YAG laser), spectrometer (1/2 m model 500 SpectraPro Acton Research Corporation) and intensified linear diode array (model 1460 Princeton Applied Research detector/controller optical multichannel analyzer). The captured time-resolved data, averaged over 100 individual LIOB events, were detector-noise/background corrected, wavelength and detector sensitivity calibrated. Further details of the experimental procedures are summarized in [3].

Individual profiles of the Balmer series lines $\mathrm{H}$-alpha, $\mathrm{H}$ beta, and H-gamma were measured subsequent to optical breakdown. Comparison of the recorded intensities of these lines allows one, in principle, to infer electron temperature, $T_{e}$, provided that reasonable complete profiles are recorded. Typical for our LIOB measurements however are incomplete $\mathrm{H}$-beta and $\mathrm{H}$-gamma line profiles due to significant contribution of background radiation early in the methane breakdown, high electron number density, $N_{e}$, early in the plasma decay followed by occurrence of molecular spectra that overlap Balmer series lines, particularly from $C_{2}$.

Temperature estimates are inferred by using the relative signal-strengths of the three line profiles $\mathrm{H}$-alpha, $\mathrm{H}$-beta, and H-gamma, although several errors in determining $T_{e}$ are noted. For example, determination of background radiation 
TABLE 1: Measured H-alpha widths.

\begin{tabular}{lcc}
\hline$t_{\text {delay }}[\mu \mathrm{s}]$ & $\begin{array}{c}2.7 \times 10^{5} \mathrm{~Pa}: \\
\text { width [nm] }\end{array}$ & $\begin{array}{c}6.5 \times 10^{5} \mathrm{~Pa}: \\
\text { width }[\mathrm{nm}]\end{array}$ \\
\hline 0.4 & $2.8 \pm 0.3$ & $2.7 \pm 0.3$ \\
0.5 & $2.4 \pm 0.3$ & $2.3 \pm 0.3$ \\
0.6 & $2.1 \pm 0.3$ & $2.1 \pm 0.3$ \\
0.7 & $1.9 \pm 0.2$ & $1.9 \pm 0.2$ \\
0.8 & $1.7 \pm 0.2$ & $1.7 \pm 0.2$ \\
0.9 & $1.6 \pm 0.2$ & $1.5 \pm 0.2$ \\
1.0 & $1.4 \pm 0.2$ & $1.4 \pm 0.2$ \\
1.1 & $1.3 \pm 0.2$ & $1.3 \pm 0.2$ \\
1.2 & $1.2 \pm 0.1$ & $1.2 \pm 0.2$ \\
1.3 & $1.1 \pm 0.1$ & $1.1 \pm 0.1$ \\
1.4 & $1.0 \pm 0.1$ & $1.0 \pm 0.1$ \\
1.5 & $1.0 \pm 0.1$ & $0.92 \pm 0.1$ \\
1.6 & $0.89 \pm 0.1$ & $0.87 \pm 0.1$ \\
1.7 & $0.86 \pm 0.1$ & $0.78 \pm 0.1$ \\
1.8 & $0.79 \pm 0.1$ & $0.77 \pm 0.1$ \\
1.9 & $0.77 \pm 0.1$ & $0.69 \pm 0.1$ \\
2.0 & $0.73 \pm 0.1$ & $0.67 \pm 0.1$ \\
2.1 & $0.70 \pm 0.1$ & $0.61 \pm 0.1$ \\
\hline
\end{tabular}

at a particular time delay from LIOB, or application of sensitivity and wavelength calibrations, or comparison of line-profiles from separate 100-event averages recorded for only one particular Balmer line at a time.

The results for the inferred $T_{e}$ are in the $10000 \mathrm{~K}$ (time delay $2.1 \mu \mathrm{s}$ ) to $20000 \mathrm{~K}$ (time delay of $0.4 \mu \mathrm{s}$ ) range, using the area of the incomplete $\mathrm{H}$-beta and $\mathrm{H}$-gamma lines and the area of the $\mathrm{H}$-alpha line in constructing Boltzmann plots, analogous as discussed in [1]. For both gas pressures of $2.7 \times$ $10^{5} \mathrm{~Pa}$ and $6.5 \times 10^{5} \mathrm{~Pa}$, almost identical results are found for $T_{e}$. When extending the curve fitting beyond the measured spectral windows used for the three lines, and using the area of these extended profiles, typically $50 \%$ higher $T_{e}$ is found: these temperatures constitute an upper limit of $15000 \mathrm{~K}$ and $30000 \mathrm{~K}$ for time delays of $2.1 \mu \mathrm{s}$ and $0.4 \mu \mathrm{s}$, respectively, with similar results obtained for both pressures.

Tables 1, 2, and 3 show measured FWHM of the Halpha, H-beta, and $\mathrm{H}$-gamma lines, respectively, for two different gas pressures: $2.7 \times 10^{5} \mathrm{~Pa}$ and $6.5 \times 10^{5} \mathrm{~Pa}$. The data are presented in tabular form for different time delays, $t_{\text {delay }}$, from LIOB. The experimental error bars indicate the estimated total error of determining the FWHM line widths for the Balmer series lines.

\section{Analysis}

The combined contribution to the FWHM of the instrumental and Doppler broadening (as well as of the fine structure) is negligibly small compared to the measured widths. For example, for the $\mathrm{H}$-gamma line those combined contributions do not exceed $0.14 \mathrm{~nm}$ for $t_{\text {delay }}=0.4 \mu \mathrm{s}$, when the experimental FWHM is at least $10.4 \mathrm{~nm}$, and do not exceed $0.11 \mathrm{~nm}$ for $t_{\text {delay }}=2.1 \mu \mathrm{s}$, when the experimental
TABLE 2: Measured H-beta widths.

\begin{tabular}{lcc}
\hline$t_{\text {delay }}[\mu \mathrm{s}]$ & $\begin{array}{c}2.7 \times 10^{5} \mathrm{~Pa}: \\
\text { width }[\mathrm{nm}]\end{array}$ & $\begin{array}{c}6.5 \times 10^{5} \mathrm{~Pa}: \\
\text { width }[\mathrm{nm}]\end{array}$ \\
\hline 0.4 & $10.0 \pm 0.9$ & $10.0 \pm 0.5$ \\
0.5 & $9.0 \pm 0.8$ & $8.7 \pm 0.5$ \\
0.6 & $8.1 \pm 0.7$ & $7.8 \pm 0.5$ \\
0.7 & $7.4 \pm 0.5$ & $7.3 \pm 0.4$ \\
0.8 & $6.8 \pm 0.4$ & $6.5 \pm 0.4$ \\
0.9 & $6.3 \pm 0.4$ & $5.9 \pm 0.4$ \\
1.0 & $5.9 \pm 0.4$ & $5.4 \pm 0.4$ \\
1.1 & $5.3 \pm 0.4$ & $5.1 \pm 0.3$ \\
1.2 & $5.0 \pm 0.3$ & $4.8 \pm 0.3$ \\
1.3 & $4.7 \pm 0.3$ & $4.5 \pm 0.3$ \\
1.4 & $4.4 \pm 0.3$ & $4.1 \pm 0.3$ \\
1.5 & $4.1 \pm 0.3$ & $3.9 \pm 0.3$ \\
1.6 & $3.9 \pm 0.3$ & $3.7 \pm 0.2$ \\
1.7 & $3.8 \pm 0.3$ & $3.5 \pm 0.2$ \\
1.8 & $3.6 \pm 0.2$ & $3.3 \pm 0.2$ \\
1.9 & $3.3 \pm 0.2$ & $3.1 \pm 0.2$ \\
2.0 & $3.1 \pm 0.2$ & $2.9 \pm 0.2$ \\
2.1 & $2.9 \pm 0.2$ & $2.8 \pm 0.2$ \\
\hline
\end{tabular}

TABle 3: Measured H-gamma widths.

\begin{tabular}{lcc}
\hline$t_{\text {delay }}[\mu \mathrm{s}]$ & $\begin{array}{c}2.7 \times 10^{5} \mathrm{~Pa}: \\
\text { width }[\mathrm{nm}]\end{array}$ & $\begin{array}{c}6.5 \times 10^{5} \mathrm{~Pa}: \\
\text { width }[\mathrm{nm}]\end{array}$ \\
\hline 0.4 & $11.0 \pm 2.0$ & $10.4 \pm 2.0$ \\
0.5 & $10.7 \pm 2.0$ & $9.5 \pm 2.0$ \\
0.6 & $8.7 \pm 1.5$ & $8.6 \pm 1.5$ \\
0.7 & $8.1 \pm 1.5$ & $7.8 \pm 1.5$ \\
0.8 & $7.6 \pm 1.5$ & $6.7 \pm 1.5$ \\
0.9 & $6.7 \pm 1.5$ & $6.3 \pm 1.5$ \\
1.0 & $6.2 \pm 1.5$ & $6.2 \pm 1.5$ \\
1.1 & $6.0 \pm 1.0$ & $5.8 \pm 1.0$ \\
1.2 & $5.8 \pm 1.0$ & $5.5 \pm 1.0$ \\
1.3 & $5.6 \pm 1.0$ & $5.4 \pm 1.0$ \\
1.4 & $5.3 \pm 1.0$ & $5.1 \pm 1.0$ \\
1.5 & $5.2 \pm 1.0$ & $5.1 \pm 1.0$ \\
1.6 & $5.0 \pm 1.0$ & $4.7 \pm 1.0$ \\
1.7 & $5.0 \pm 1.0$ & $5.0 \pm 1.0$ \\
1.8 & $5.0 \pm 1.0$ & $4.6 \pm 1.0$ \\
1.9 & $5.0 \pm 1.0$ & $4.4 \pm 1.0$ \\
2.0 & $4.7 \pm 1.0$ & $3.6 \pm 1.0$ \\
2.1 & $4.6 \pm 1.0$ & $3.6 \pm 1.0$ \\
\hline & &
\end{tabular}

FWHM is at least $3.6 \mathrm{~nm}$. A similar situation is for the $\mathrm{H}$ beta and $\mathrm{H}$-alpha lines-except for few largest time delays for the $\mathrm{H}$-alpha line, where those contributions reach up to about $15 \%$ of the measured FWHM and should be taken into account.

For each of these three hydrogen lines, for 18 time delays and 2 different gas pressures the values of the electron density $N_{e}$ were deduced based on the Stark broadening tables and analytical results from $[4,5]$. Table 4 shows the values of $N_{e}$ 
TAble 4: Deduced $N_{e}$ for pressure of $2.7 \times 10^{5} \mathrm{~Pa}$.

\begin{tabular}{lccc}
\hline$t_{\text {delay }}[\mu \mathrm{s}]$ & $\begin{array}{c}H_{\alpha} \\
N_{e}\left[10^{17} \mathrm{~cm}^{-3}\right]\end{array}$ & $\begin{array}{c}H_{\beta} \\
N_{e}\left[10^{17} \mathrm{~cm}^{-3}\right]\end{array}$ & $\begin{array}{c}H_{\gamma} \\
N_{e}\left[10^{17} \mathrm{~cm}^{-3}\right]\end{array}$ \\
\hline 0.4 & $5.0 \pm 0.4$ & $2.9 \pm 0.8$ & $2.6 \pm 0.7$ \\
0.5 & $3.8 \pm 0.4$ & $2.5 \pm 0.7$ & $2.5 \pm 0.7$ \\
0.6 & $3.0 \pm 0.4$ & $2.2 \pm 0.6$ & $1.9 \pm 0.5$ \\
0.7 & $2.5 \pm 0.4$ & $1.9 \pm 0.5$ & $1.7 \pm 0.5$ \\
0.8 & $2.1 \pm 0.3$ & $1.6 \pm 0.4$ & $1.5 \pm 0.5$ \\
0.9 & $1.9 \pm 0.3$ & $1.4 \pm 0.4$ & $1.3 \pm 0.4$ \\
1.0 & $1.6 \pm 0.3$ & $1.3 \pm 0.4$ & $1.2 \pm 0.4$ \\
1.1 & $1.4 \pm 0.2$ & $1.2 \pm 0.3$ & $1.1 \pm 0.25$ \\
1.2 & $1.3 \pm 0.2$ & $1.1 \pm 0.3$ & $1.05 \pm 0.25$ \\
1.3 & $1.15 \pm 0.2$ & $1.0 \pm 0.3$ & $1.0 \pm 0.28$ \\
1.4 & $1.0 \pm 0.2$ & $0.87 \pm 0.3$ & $0.95 \pm 0.28$ \\
1.5 & $1.0 \pm 0.2$ & $0.79 \pm 0.3$ & $0.92 \pm 0.28$ \\
1.6 & $0.90 \pm 0.2$ & $0.76 \pm 0.2$ & $0.90 \pm 0.28$ \\
1.7 & $0.84 \pm 0.1$ & $0.75 \pm 0.2$ & $0.90 \pm 0.29$ \\
1.8 & $0.72 \pm 0.1$ & $0.68 \pm 0.2$ & $0.90 \pm 0.29$ \\
1.9 & $0.69 \pm 0.1$ & $0.60 \pm 0.2$ & $0.90 \pm 0.29$ \\
2.0 & $0.63 \pm 0.1$ & $0.55 \pm 0.2$ & $0.80 \pm 0.30$ \\
2.1 & $0.58 \pm 0.1$ & $0.50 \pm 0.2$ & $0.77 \pm 0.29$ \\
\hline
\end{tabular}

TABle 5: Deduced $N_{e}$ for pressure of $6.5 \times 10^{5} \mathrm{~Pa}$.

\begin{tabular}{lccc}
\hline$t_{\text {delay }}[\mu \mathrm{s}]$ & $\begin{array}{c}H_{\alpha} \\
N_{e}\left[10^{17} \mathrm{~cm}^{-3}\right]\end{array}$ & $\begin{array}{c}H_{\beta} \\
N_{e}\left[10^{17} \mathrm{~cm}^{-3}\right]\end{array}$ & $\begin{array}{c}H_{\gamma} \\
N_{e}\left[10^{17} \mathrm{~cm}^{-3}\right]\end{array}$ \\
\hline 0.4 & $4.9 \pm 0.8$ & $2.9 \pm 0.8$ & $2.4 \pm 0.7$ \\
0.5 & $3.6 \pm 0.7$ & $2.4 \pm 0.7$ & $2.2 \pm 0.6$ \\
0.6 & $3.0 \pm 0.6$ & $2.1 \pm 0.6$ & $1.8 \pm 0.5$ \\
0.7 & $2.5 \pm 0.5$ & $1.8 \pm 0.5$ & $1.6 \pm 0.5$ \\
0.8 & $2.1 \pm 0.4$ & $1.5 \pm 0.4$ & $1.3 \pm 0.4$ \\
0.9 & $1.75 \pm 0.4$ & $1.3 \pm 0.4$ & $1.2 \pm 0.4$ \\
1.0 & $1.6 \pm 0.4$ & $1.2 \pm 0.4$ & $1.2 \pm 0.4$ \\
1.1 & $1.4 \pm 0.3$ & $1.1 \pm 0.3$ & $1.05 \pm 0.25$ \\
1.2 & $1.3 \pm 0.3$ & $1.0 \pm 0.3$ & $1.0 \pm 0.26$ \\
1.3 & $1.15 \pm 0.3$ & $0.92 \pm 0.3$ & $1.0 \pm 0.28$ \\
1.4 & $1.0 \pm 0.3$ & $0.79 \pm 0.3$ & $0.91 \pm 0.28$ \\
1.5 & $0.92 \pm 0.3$ & $0.76 \pm 0.3$ & $0.91 \pm 0.28$ \\
1.6 & $0.86 \pm 0.3$ & $0.72 \pm 0.3$ & $0.78 \pm 0.27$ \\
1.7 & $0.70 \pm 0.3$ & $0.66 \pm 0.3$ & $0.90 \pm 0.29$ \\
1.8 & $0.69 \pm 0.3$ & $0.60 \pm 0.3$ & $0.77 \pm 0.28$ \\
1.9 & $0.57 \pm 0.3$ & $0.55 \pm 0.3$ & $0.71 \pm 0.28$ \\
2.0 & $0.55 \pm 0.4$ & $0.50 \pm 0.4$ & $0.51 \pm 0.25$ \\
2.1 & $0.46 \pm 0.4$ & $0.48 \pm 0.4$ & $0.51 \pm 0.25$ \\
\hline
\end{tabular}

deduced from these three hydrogen lines for the pressure $2.7 \times 10^{5} \mathrm{~Pa}$; Table 5 shows the values of $N_{e}$ deduced from these three hydrogen lines for the pressure $6.5 \times 10^{5} \mathrm{~Pa}$.

The error bars of the deduced $N_{e}$ are due to several factors as follows. The primary factor is the error bars of the experimental widths. There are two secondary factors: the uncertainty in the temperature and the uncertainty in the reduced mass of the pairs "perturber-radiator". Speaking of the latter, since the discharge occurs in methane $(\mathrm{CH} 4)$, the perturbers could be not only hydrogen ions (protons) but also carbon ions. The reduced mass is $\mu=0.5$ for the pairs $\mathrm{H}-\mathrm{H}+$ or $\mu=0.923$ for the pairs $\mathrm{H}-\mathrm{C}+$ and $\mathrm{H}-\mathrm{C}++$.

\section{Conclusions}

We demonstrated that time-resolved measurements of profiles of $\mathrm{H}$-alpha, $\mathrm{H}$-beta, and $\mathrm{H}$-gamma lines provide good diagnostics for the LIOB in methane.

The electron densities $N_{e}$ deduced from the H-gamma and $\mathrm{H}$-beta lines showed generally a good agreement with each other. At $t_{\text {delay }}=0.4 \mu \mathrm{s}$ and $t_{\text {delay }}=0.5 \mu \mathrm{s}$, the electron density $N_{e}$ formally deduced from the $\mathrm{H}$-alpha line exceeds $\mathrm{N}_{e}$ deduced from the H-beta and $\mathrm{H}$-gamma lines beyond the error bars (while $N_{e}$ deduced from the H-beta and $\mathrm{H}$ gamma lines agree with each other very well). This is a clear indication that at $t_{\text {delay }}=0.4 \mu \mathrm{s}$ and $t_{\text {delay }}=0.5 \mu \mathrm{s}$, the $\mathrm{H}$ alpha line experienced a significant self-absorption.

Besides, for the range of $t_{\text {delay }}=0.6-1.2 \mu \mathrm{s}$, the Mean Probable Values (MPVs) of $N_{e}$ formally deduced from the $\mathrm{H}$-alpha line significantly exceed MPV of $N_{e}$ deduced from the $\mathrm{H}$-beta and $\mathrm{H}$-gamma lines (though the disagreement is within the error bars). Thus it is probable that at $t_{\text {delay }}=0.6-$ $1.2 \mu \mathrm{s}$, the $\mathrm{H}$-alpha still experienced some self-absorption.

We mention that if we would have used the Stark broadening diagnosis-map data from [6], we would have deduced slightly different electron densities. This is because the computational results of paper [6] are based on a simulation model that neglects the direct coupling of the electron and ion microfields taken into account analytically in $[4,5]$.

We note that for a particular hydrogen line and for a specific time delay, our experimental widths for the higher pressure are generally lower than for the lower pressure. However, generally the higher the pressure is, the higher the expected electron density is. So, there should be some additional broadening mechanism that is more effective at the lower density than at the higher density. Such mechanism could be electrostatic plasma turbulence, that is, some regular or stochastic electrostatic waves in plasmas (see, e.g., $[7,8]$ and references therein).

Electrostatic waves produce an additional Stark broadening controlled by their amplitude. The collisional damping of electrostatic waves increases with the density, so that their amplitude and the additional Stark broadening decreases with the density.

A future study should analyze this hypothesis in more detail or provide an alternative explanation.

\section{References}

[1] C. G. Parigger, D. H. Plemmons, and J. W. L. Lewis, "Spatially and Temporally Resolved Electron Number Density Measurements in a Decaying Laser-Induced Hydrogen Plasma Using Hydrogen-Alpha Line Profiles," Applied Optics, vol. 34, p. 3325, 1995.

[2] C. G. Parigger, D. H. Plemmons, and E. Oks, "Balmer series $\mathrm{H}_{\beta}$ measurements in a laser-induced hydrogen plasma," Applied Optics, vol. 42, no. 30, pp. 5992-6000, 2003. 
[3] C. G. Parigger, M. Dackman, and J. O. Hornkohl, "Timeresolved spectroscopy measurements of hydrogen-alpha, -beta, and -gamma emissions," Applied Optics, vol. 47, no. 31, pp. G1G6, 2008.

[4] E. Oks, Stark Broadening of Hydrogen and Hydrogenlike Spectral Lines in Plasmas: The Physical Insight, Alpha Science International, Oxford, UK, 2006.

[5] E. Oks, "Stark Widths of Hydrogen Spectral Lines in Plasmas: a Highly-Advanced Non-Simulative Semiclassical Theory and Tables," in Proceedings of the 18th International Conference on Spectral Line Shapes, vol. 874 of AIP Conference Proceedings, p. 5, American Institute of Physics, 2006.

[6] M. A. Gigosos, M. Á. González, and V. Cardeñoso, "Spectrochim," Acta B, p. 1489, 2003.

[7] E. Oks, Plasma Spectroscopy: The Influence of Microwave and Laser Fields, vol. 9 of Springer Series on Atoms and Plasmas, Springer, New York, NY, USA, 1995.

[8] H. R. Griem, Spectral Line Broadening by Plasmas, Academic Press, New York, NY, USA, 1964. 


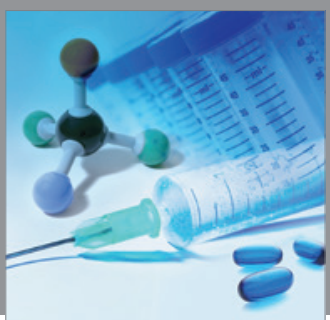

International Journal of

Medicinal Chemistry

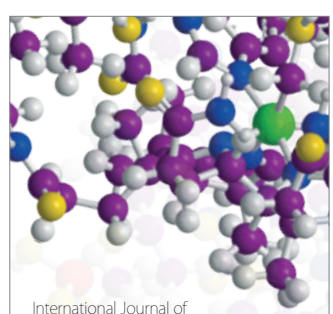

Carbohydrate Chemistry

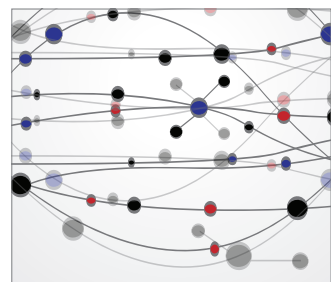

The Scientific World Journal
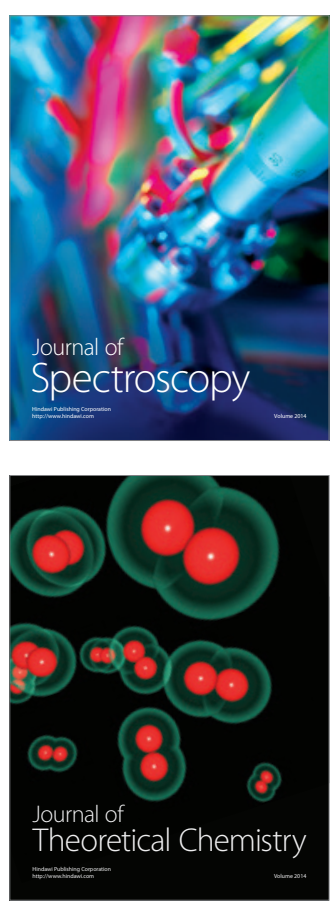
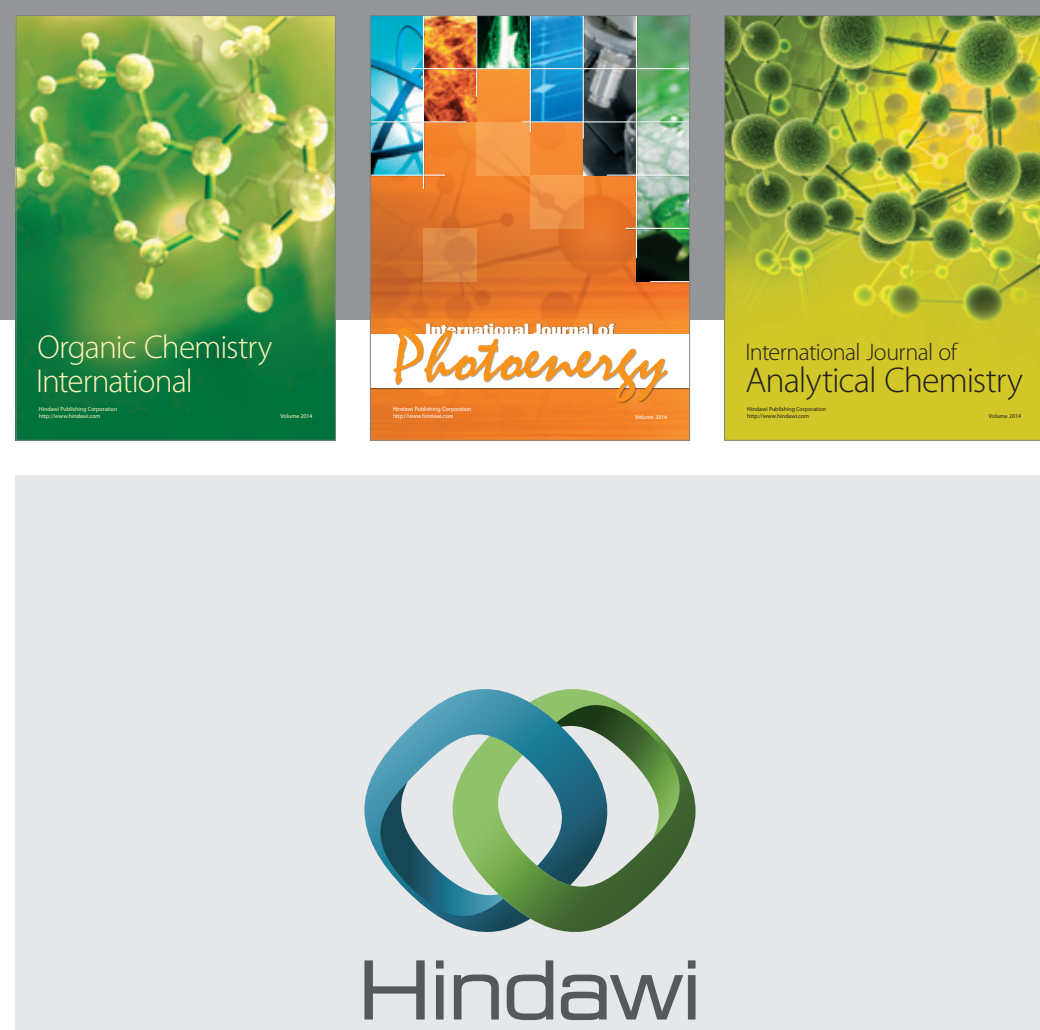

Submit your manuscripts at

http://www.hindawi.com
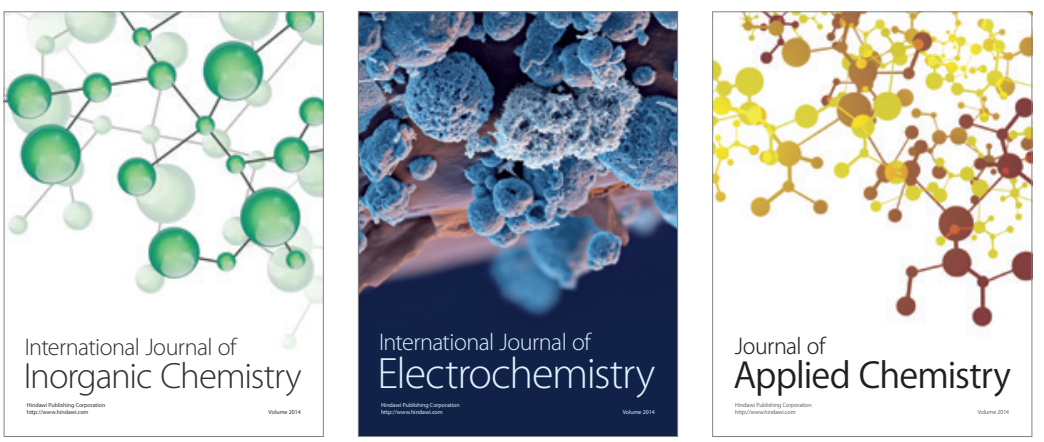

Journal of

Applied Chemistry
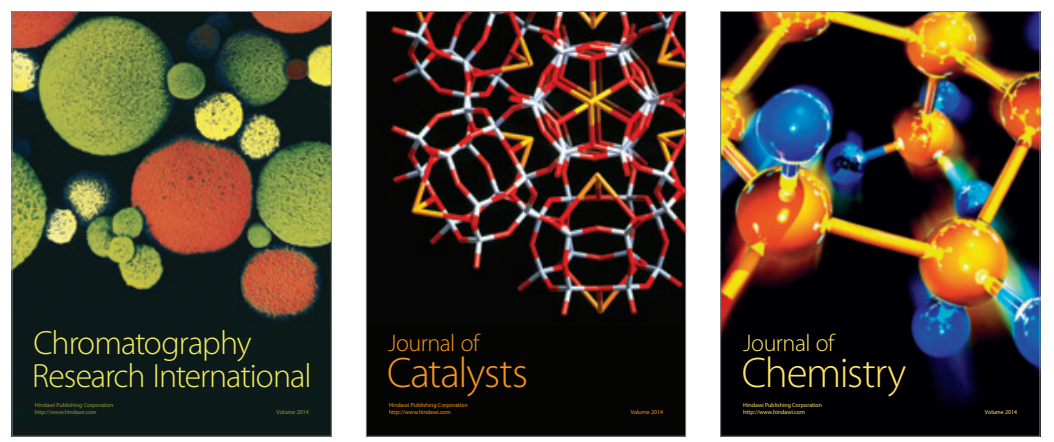
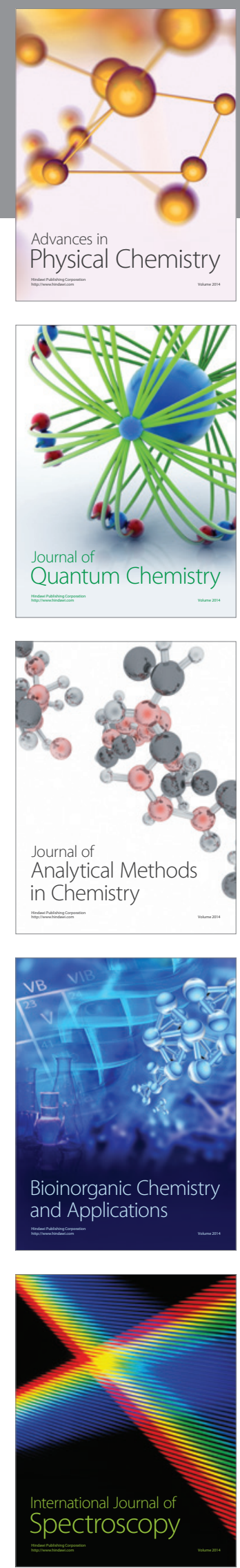